\title{
The Present State and Future Perspectives of Cardiac Regenerative Therapy Using Human Pluripotent Stem Cells
}

\author{
Yusuke Soma, Yuika Morita, Yoshikazu Kishino, Hideaki Kanazawa, Keiichi Fukuda and \\ Shugo Tohyama*
}

Department of Cardiology, Keio University School of Medicine, Tokyo, Japan

\section{OPEN ACCESS}

Edited by:

Jeehoon Kang,

Seoul National University Hospital,

South Korea

Reviewed by:

Mingtao Zhao,

Nationwide Children's Hospital,

United States

Huang-Tian Yang,

Shanghai Institute for Biological

Sciences, Chinese Academy of

Sciences (CAS), China

Sara Pahlavan,

Royan Institute for Stem Cell Biology

and Technology (RI-SCBT), Iran

*Correspondence:

Shugo Tohyama

shugotohyama@keio.jp

Specialty section:

This article was submitted to Cardiovascular Biologics and

Regenerative Medicine,

a section of the journal

Frontiers in Cardiovascular Medicine

Received: 11 September 2021

Accepted: 25 October 2021

Published: 08 December 2021

Citation:

Soma Y, Morita Y, Kishino Y,

Kanazawa H, Fukuda K and Tohyama S (2021) The Present State and Future Perspectives of Cardiac Regenerative Therapy Using Human

Pluripotent Stem Cells.

Front. Cardiovasc. Med. 8:774389.

doi: 10.3389/fcvm.2021.774389
The number of patients with heart failure (HF) is increasing with aging in our society worldwide. Patients with HF who are resistant to medication and device therapy are candidates for heart transplantation (HT). However, the shortage of donor hearts is a serious issue. As an alternative to $H T$, cardiac regenerative therapy using human pluripotent stem cells (hPSCs), such as human embryonic stem cells and induced pluripotent stem cells, is expected to be realized. Differentiation of hPSCs into cardiomyocytes (CMs) is facilitated by mimicking normal heart development. To prevent tumorigenesis after transplantation, it is important to eliminate non-CMs, including residual hPSCs, and select only CMs. Among many CM selection systems, metabolic selection based on the differences in metabolism between CMs and non-CMs is favorable in terms of cost and efficacy. Large-scale culture systems have been developed because a large number of hPSC-derived CMs (hPSC-CMs) are required for transplantation in clinical settings. In large animal models, hPSC-CMs transplanted into the myocardium improved cardiac function in a myocardial infarction model. Although post-transplantation arrhythmia and immune rejection remain problems, their mechanisms and solutions are under investigation. In this manner, the problems of cardiac regenerative therapy are being solved individually. Thus, cardiac regenerative therapy with hPSC-CMs is expected to become a safe and effective treatment for HF in the near future. In this review, we describe previous studies related to hPSC-CMs and discuss the future perspectives of cardiac regenerative therapy using hPSC-CMs.

Keywords: regenerative therapy, embryonic stem cell (ES cells), induced pluripotent stem cell (iPS cell) (iPSC), stem cell metabolism, heart failure

\section{INTRODUCTION}

The incidence of heart failure (HF) is high in the aged population, which is growing at a rapid pace worldwide. HF patients with who are resistant to optimal medication and device therapy are candidates for heart transplantation (HT). However, the number of HTs is $<100$ per year in Japan, and the mean waiting period is $\sim 4$ years (1). Cardiac regenerative therapy using human pluripotent stem cells (hPSCs) such as human embryonic stem cells (hESCs) and human induced pluripotent stem cells (hiPSCs) is expected to become an alternative to HT for patients with severe HF (2-4). 
A large number of hPSC-derived cardiomyocytes (hPSCCMs) are required for cardiac regenerative therapy (5). Many studies have revealed that differentiation of hPSCs into cardiomyocytes (CMs) can be induced by imitating normal heart development, particularly by controlling developmental signals such as Wnt and BMP signal (6-11). Moreover, in recent years, the following issues have been explored, and many possible solutions have been discovered: (1). Elimination of non-CMs, including undifferentiated hPSCs, and selection of hPSC-CMs to prevent tumorigenesis after transplantation, (2). Large-scale cultures of hPSCs and hPSC-CMs, (3). Effective transplantation of hPSC-CMs into host myocardium (Figure 1A). The following issues remain, and further research is necessary: (1). Arrhythmia after transplantation of hPSC-CMs and (2). Immune rejection. We will discuss these issues and the studies that have been conducted to solve them.

\section{ELIMINATION OF UNDIFFERENTIATED HPSCS AND SELECTION OF HPSC-CMS}

When hPSC-CMs are transplanted into the host myocardium, residual non-CMs, including undifferentiated hPSCs, are a problem because these cells can induce tumors such as teratomas. Even if the contamination of undifferentiated hPSCs spiked into non-tumorigenic cells is $\sim 0.025 \%$, teratomas may be generated if these cells are injected into the legs of immunodeficient mice (12). To solve this problem, methods to eliminate undifferentiated hPSCs or to select only hPSC-CMs have been developed (Table 1A) (13-28).

\section{Cell Sorting}

The methods using fluorescence-activated cell sorting (FACS) or magnetic cell sorting (MACS) are reasonable. Antibodies against pluripotent markers such as TRA-1-60, SSEA-4, and SSEA-5 on hPSCs enable their removal $(13,14)$. In addition, signalregulatory protein alpha (SIRPA) is expressed specifically on hPSC-CMs, and an antibody against SIRPA can select hPSCCMs (16). However, since cardiac regenerative therapy requires a large number of CMs, cell sorting is not favorable because it requires considerable effort and time. Therefore, other methods have been developed.

\section{Addition of Small-Molecule Compounds}

Chemical treatment is a fast and inexpensive method for eliminating undifferentiated hPSCs. For example, the addition of a recombinant lectin-toxin fusion protein that combines with hPSCs eliminates them selectively (20). Additionally, the newly designed phospho-D-peptide, D-3, rapidly induces toxicity in hPSCs via alkaline phosphatases and can eliminate them (18).

\section{Metabolic Regulation}

Metabolic regulation affects cell fate and is a costeffective method for eliminating undifferentiated hPSCs or selecting hPSC-CMs. Methionine and its metabolite, S-adenosylmethionine (SAM), are key regulators of hPSC maintenance and differentiation. Short-term methionine deprivation results in a rapid decrease in intracellular SAM levels, triggering the activation of p53-p38 signaling, reducing NANOG expression, and poising hPSCs for differentiation. Moreover, prolonged methionine deprivation induces hPSC apoptosis and elimination (24).

Elimination of residual proliferating cells, including undifferentiated hPSCs, is effective in preventing tumorigenesis. We focused on the differences in metabolism between CMs and non-CMs. Glycolysis is enhanced in proliferating cells, including undifferentiated hPSCs, even under sufficient oxygen conditions. They produce energy by glycolysis, and they also produce nucleic acids, amino acids, and lipids, which are required for cell proliferation. In contrast, hPSC-CMs produce energy effectively using the tricarboxylic acid (TCA) cycle and fatty acid oxidation. In addition, unlike hPSCs, hPSC-CMs can use lactate as an energy source. Therefore, we selected only hPSC-CMs by culturing them in glucose-free medium supplemented with lactate (22).

Next, we focused on amino acid metabolism. We discovered that hPSCs use glutamine in addition to glucose in the TCA cycle. Using this characteristic of hPSCs, we selected hPSC-CMs more effectively in glucose- and glutamine-free media supplemented with lactate (23). In addition, this method promotes metabolic maturation of hPSC-CMs (36). This means that the hPSC-CMs surviving in glucose-free medium supplemented with lactate alter their metabolism from glycolysis to lactate oxidation, which is similar to the metabolic change in heart development from fetal to neonatal state $(37,38)$. hPSC-CMs that have undergone this metabolic selection are likely to engraft and become mature when transplanted into the ischemic region where lactate accumulates (39) (Figure 1B).

Lipid metabolism is also important for hPSCs. An inhibitor of stearoyl-CoA desaturase, the key enzyme in oleic acid biosynthesis, induces endoplasmic reticulum (ER) stress and apoptosis in hPSCs (25). Moreover, we revealed that fatty acid synthesis is important for hPSC survival and Orlistat, which inhibits fatty acid synthase, can safely induce cell death in hPSCs via mitochondria-mediated apoptosis (26).

\section{LARGE-SCALE CULTURE OF HPSCS AND HPSC-CMS}

The human left ventricle contains approximately four billion cardiomyocytes $(5,40)$. It has been reported that $\sim 25 \%$ loss of cardiomyocytes due to infarction causes HF (41), and it is necessary to assemble over one billion cardiomyocytes for cardiac regenerative therapy $(5,41)$. There are mainly two methods available to produce these many hPSC-CMs (Table 1B) (29-35).

\section{Three-Dimensional Culture}

Three-dimensional (3D) suspension culture using a large bioreactor or spinner flask is suitable for the large-scale hPSCs culture. The advantage of $3 \mathrm{D}$ culture is that it does not require expensive cell-adhesive coating proteins, such as vitronectin and laminin. Moreover, 3D cultures can be easily harvested from medium. In some studies, microcarriers were used in 


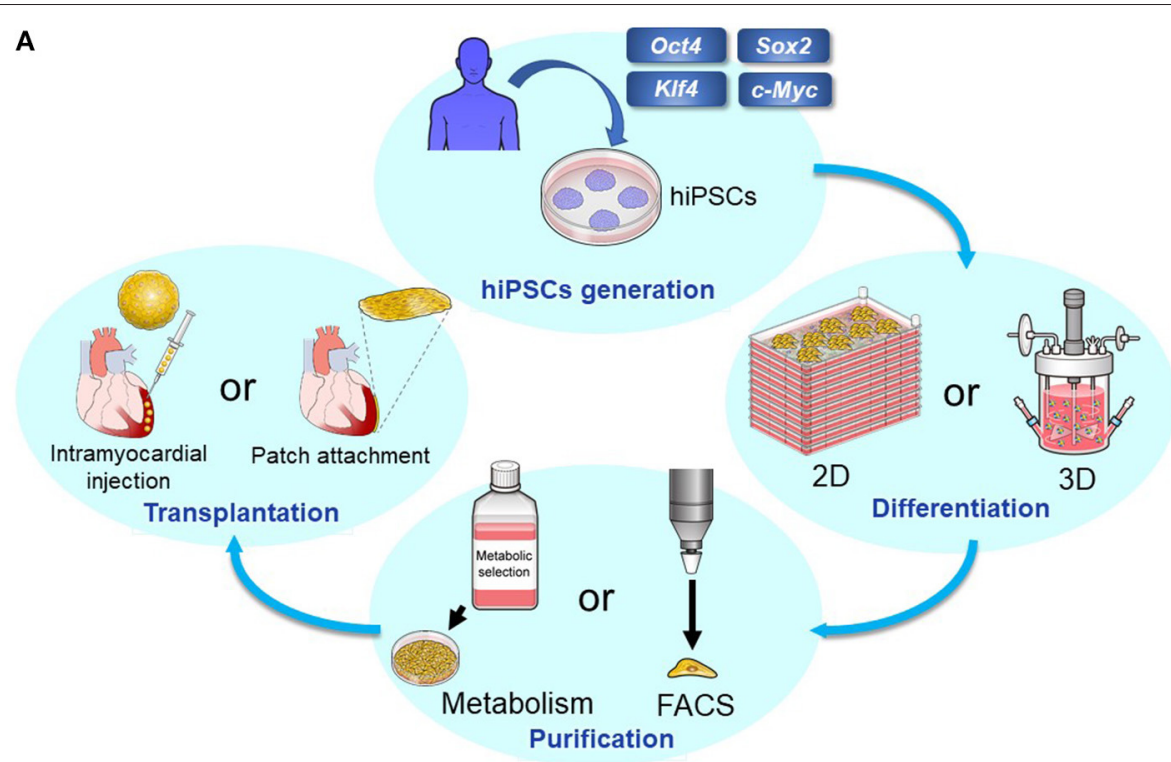

B

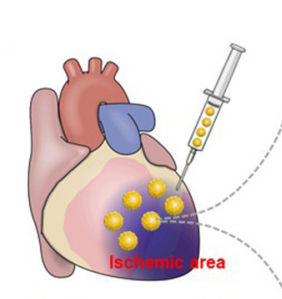

Lactate accumulation
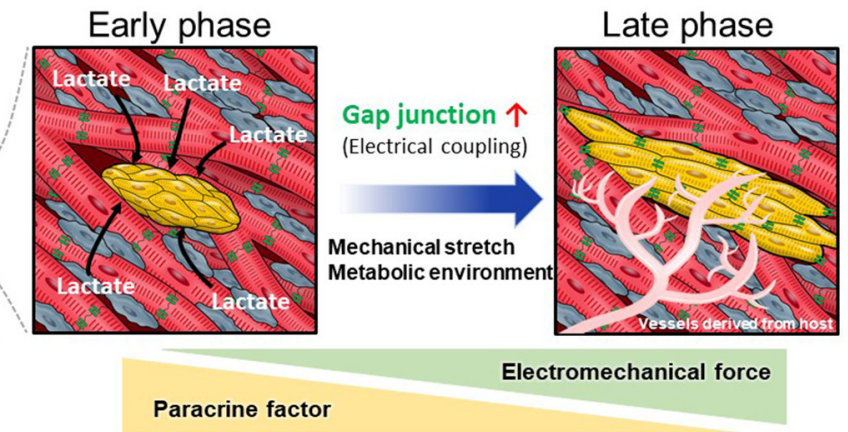

FIGURE 1 | (A) The scheme of cardiac regenerative therapy. hPSCs were established by introducing transcription factors including Oct4, Sox2, Klf4, and c-Myc to human somatic cells. Two-dimensional or three-dimensional large-scale culture systems for hPSCs and hPSC-CMs have been developed. Using these systems, we can culture a large number of hPSCs and induce their differentiation into CMs effectively. Elimination of non-CMs including undifferentiated hPSCs is important to prevent tumorigenesis. Particularly, metabolic selection is useful and cost-effective. Then, we transplant hPSC-CMs into the host myocardium. There are two main methods. One method is to transplant hPSC-CMs as a patch onto the host epicardium. The other method is to inject them using a needle into the host myocardium. (B) Intramyocardial transplantation of metabolically selected cardiomyocytes. hPSC-CMs that have undergone the metabolic selection in glucose- and glutamine-free medium supplemented with lactate are likely to engraft and become mature when they are transplanted into the ischemic region because of lactate accumulation.

There is a high density of host-derived microvessels within the graft, which promotes the engraftment and maturation of transplanted hPSC-CMs.

combination with $3 \mathrm{D}$ suspension culture, and were used as a matrix for floating cells $(33,34)$. This is why many studies focus more on 3D suspension large-scale cultures (29-34). For example, Chen et al. reported that, using 3D suspension culture, they could consistently differentiate hPSCs to $>90 \%$ $\mathrm{CM}$ purity with an average yield of $\sim 1.5-2 \times 10^{9} \mathrm{CMs} / \mathrm{L}$ using $1 \mathrm{~L}$ spinner flasks after 25 days of differentiation. They achieved this by modulating the Wnt pathway and optimizing cell aggregate size, small molecule concentrations, induction timing, and agitation rate (29). Halloin et al. applied stirred bioreactor systems and uninterrupted chemical Wnt pathway control during the early stages of differentiation. They obtained yields of $\sim 1 \times 10^{6} \mathrm{CMs} / \mathrm{mL}$, ultimately resulting in the production of $\sim 4.0 \times 10^{8} \mathrm{CMs}$ at $>90 \%$ lineage purity after 10 days of differentiation (32).

\section{Two-Dimensional Culture}

The advantage of $2 \mathrm{D}$ culture is that cells are evenly exposed to the culture medium and reagents. Because differentiation of hPSCs into CMs and metabolic selection of non-CMs are more effective in 2D culture than in 3D culture, we established a largescale $2 \mathrm{D}$ culture system by stacking 10 culture plates $(35,42)$. Under normal culture conditions, the proliferation of hPSCs was not stable because the $\mathrm{CO}_{2}$ concentration increased and the $\mathrm{O}_{2}$ concentration decreased in the medium. Using an active gas ventilation system with $5 \% \mathrm{CO}_{2}$, we could produce $\sim 1.2 \times 10^{9}$ CMs at $\sim 80 \%$ purity per culture and increase the purity to $>97 \%$ using metabolic selection (35).

Cell-adhesive coating proteins such as vitronectin and laminin are used for 2D culture instead of feeder cells $(43,44)$; however, they are expensive and may become an obstacle in 
TABLE 1 | Overview of the main studies investigating (A) elimination of undifferentiated hPSCs or selection of hPSC-CMs and (B) large-scale culture of hPSCs and hPSC-CMs.

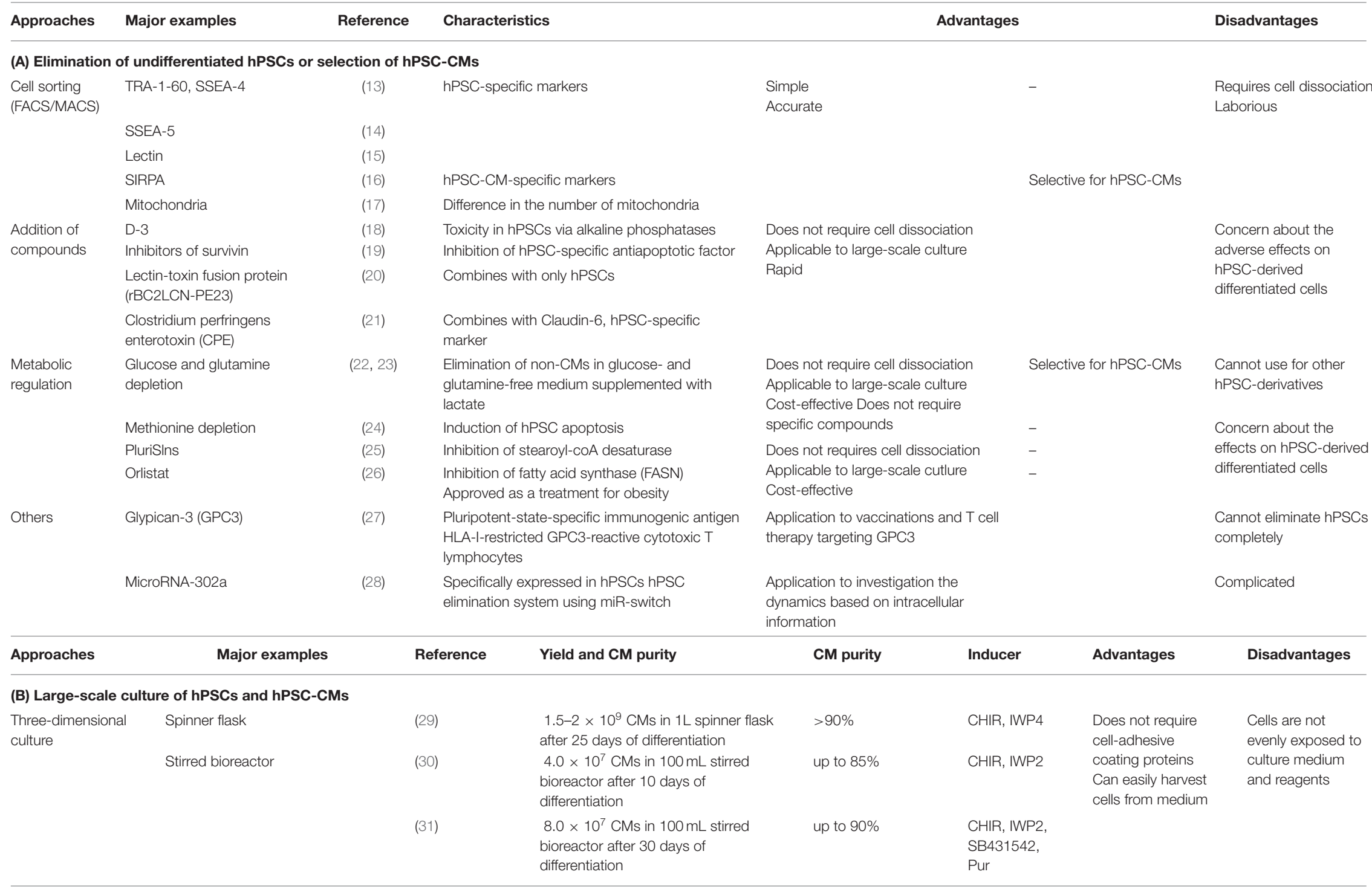




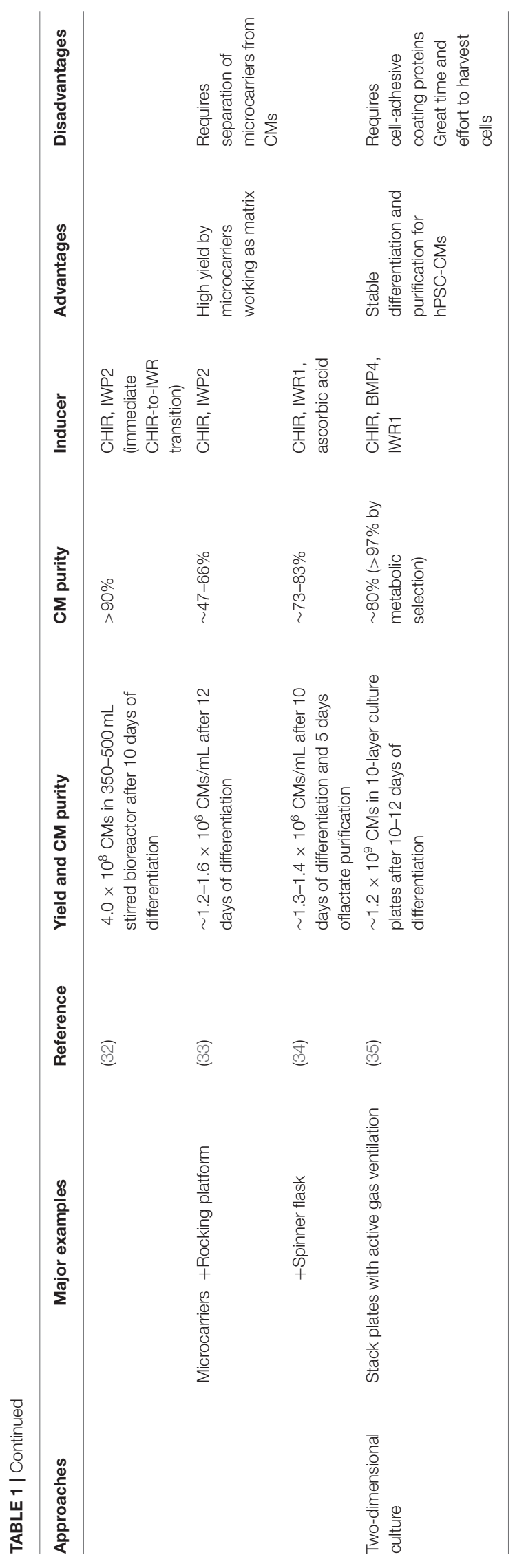

clinical settings. It has been reported that artificially synthesized polymer-based coatings are cost-effective for the adhesion of hPSCs $(45,46)$.

To produce large number of hPSCs more cost-effectively, we found that tryptophan (TRP) plays a key role in the proliferation and maintenance of pluripotency. We produced a large number of hPSCs in TRP-supplemented medium in combination with a large-scale 2D culture system (47).

In conclusion, both $3 \mathrm{D}$ and $2 \mathrm{D}$ cultures could yield a large number of hPSC-CMs required for clinical application, although each method has its advantages and disadvantages.

\section{THE METHOD FOR TRANSPLANTING HPSC-CMS}

There are two main methods for the transplantation of hPSCCMs into the host myocardium. One method is to transplant hPSC-CMs as a patch on the surface of the host heart. The other method involves injecting hPSC-CMs into the host myocardium using a needle.

\section{hPSC-CM Patch Attachment}

The advantage of transplanting hPSC-CMs as a patch or sheet is that operators can visually confirm attachment to the host heart. Also, the damage to the host myocardium by patch attachment is less than that by the direct injection method. In contrast, hPSCCM patches transplanted onto the epicardium tended to drop out in a relatively short time. For example, Kawamura et al. reported that transplantation of hPSC-CM sheets to infarcted porcine hearts improved cardiac performance; however, but after 8 weeks after transplantation, very few hPSC-CMs survived (48). Therefore, the reason for the improvement in cardiac function was thought to be mainly due to paracrine effects of cytokines and growth factors secreted by the sheets rather than the direct contraction force. In previous research, the host heart and hPSC$\mathrm{CM}$ patches on the epicardium did not contract synchronously because the epicardium is an electrical insulator (49). In contrast, Higuchi et al. reported that, using high-flux synchrotron X-ray diffraction, hPSC-CM sheets contracted synchronously with the host heart and suggested that the integration depends on the microenvironment of native cardiac tissue and epicardium (50).

Engineered heart tissue (EHT) made from hPSC-CMs is commonly used in drug screening and disease modeling. In addition, studies using EHT for transplantation have also been reported (51-54). In general, EHT was transplanted onto the epicardium and hPSC-CM patches. For example, Li et al. obtained cardiac tissue-like constructs by cultivating hPSC-CMs on low-thickness aligned nanofibers and transplanted them into infarcted rat hearts (51). The constructs improved left ventricular function and promoted angiogenesis in the peri-ischemic zone 4 weeks after transplantation. Querdel et al. created meshstructured tissue patches consisting of hPSC-CMs. Transplanting them into infarcted guinea pig hearts improved left cardiac function 4 weeks after transplantation. They created human-scale patches consisting of $4.5 \times 10^{8} \mathrm{hPSC}-\mathrm{CMs}$. They transplanted the 
patches into pig hearts and confirmed their technical feasibility, although they were followed up for only 2 weeks (53).

\section{Intramyocardial Injection of hPSC-CMs}

The other method involves injecting hPSC-CMs using a needle into the host myocardium. Transplantation of hPSC-CMs into the border zone of the ischemic area improved left ventricular function in myocardial infarction model experiments in rodents, monkeys, and swine $(10,55-59)$. It is considered that the improvement in cardiac function is due to the direct binding between the transplanted hPSC-CMs and host CMs in addition to paracrine effects. In some studies, hPSC-CMs and host $\mathrm{CMs}$ showed synchronous electrical coupling and contraction. Moreover, hPSC-CMs transplanted into the host myocardium became mature in vivo (55-62). Although they were initially as small as fetal CMs, the transplanted hPSCCMs gradually increased in size over time. In addition, the sarcomere structure developed in a more favorable way, and isoforms of sarcomere proteins changed the mature types after transplantation. Expression of connexin 43, a gap junction protein, was increased in hPSC-CM grafts. Many factors, such as mechanical stretch, electrical stimulation, and paracrine and endocrine effects of cytokines and hormones, are thought to contribute to the maturation of hPSC-CMs in vivo. In addition, hPSC-CMs produce vascular endothelial growth factors and promote angiogenesis (59). Therefore, there was a high density of host-derived microvessels within the graft, which promoted the engraftment and maturation of transplanted hPSC-CMs.

However, even if hPSC-CMs are transplanted into single cells, the engraftment rate is very low (63). This is because transplanted hPSC-CMs flow out through the needle hole by beating the host heart. In addition, they undergo apoptosis because there is no scaffolding. Several methods have been developed to increase engraftment. For example, transplantation of cells with extracellular matrix and bioactive agents such as a growth factor, a cell-permeant peptide, a caspase inhibitor and so on was effective $(10,64,65)$. Moreover, we developed a method to transplant hPSC-derived cardiac spheroids (hPSCCSs) consisting of hPSC-CMs (66). By culturing hPSC-CMs in a microwell plate, we generated hPSC-CSs with a diameter of $150-200 \mu \mathrm{m}$, consisting of $\sim 1,000 \mathrm{hPSC}-\mathrm{CMs}$. Transplantation of hPSC-CSs into infarcted rodent hearts significantly improved the rate of engraftment and cardiac function compared to transplantation of hPSC-CMs as a single cell. Moreover, hPSCCSs also improved left ventricular function and promoted angiogenesis in infarcted swine hearts (59). Furthermore, we developed a special transplant needle (66). This needle has six side holes through which the hPSC-CSs can pass without damage. We could transplant hPSC-CSs without inducing complications such as needle perforation, hemorrhage, or tissue injury.

In recent years, it has been reported that transplanting non-CMs in addition to CMs improved cardiac function to a greater extent than transplantation of $\mathrm{CM}$ alone. For example, co-transplantation of hPSC-CMs into the myocardium and a human mesenchymal stem cell-loaded patch onto the epicardium amplified cardiac repair in infarcted rat hearts. It has been suggested that paracrine effects induced by the patch promote angiogenesis and engraftment of hPSC-CMs (67). In addition, co-transplantation of hPSC-derived epicardial cells and hPSCCMs augmented vascularization, resulting in increased graft size and improved cardiac function. hPSC-derived epicardial cells underwent epithelial-mesenchymal transition to fibroblast-like cells. They then secreted the extracellular matrix, which could contribute to cardiac repair (68). Moreover, Sun et al. reported that co-transplantation of microvessels derived from rat adipose tissue and hPSC-CMs promoted a 6-fold increase in hPSC$\mathrm{CM}$ survival and superior functional recovery in infarcted rat hearts (69). The safety and detailed mechanism by which cotransplantation of CMs and non-CMs augments cardiac repair should be analyzed in future studies.

Whether the transplantation of mature hPSC-CMs is better than that of immature hPSC-CMs remains controversial. Funakoshi et al. created mature, compact ventricular CMs from hPSCs. They compared the transplantation of mature and immature CMs in infarcted rat hearts. The transplanted mature CMs had longer sarcomeres and expressed more connexin 43 than immature CMs 8 weeks after transplantation. Therefore, transplanting mature CMs is expected to mitigate arrhythmias and lead to the development of safer therapies. In contrast, the graft size of immature CMs was larger than that of mature CMs because immature CMs had a stronger ability to proliferate (70).

In almost all studies of hPSC-CM transplantation, experimental animals were followed up for up to 3 months. Therefore, the long-term effects of transplantation remain unknown. Moreover, they used myocardial infarction models because it is difficult to create a dilated cardiomyopathy model. These issues should be addressed when cardiac regenerative therapy is applied in clinical settings. Although the methods of transplanting cells by trans-catheter intracoronary infusion or injection from the endocardial side have been mainly applied for the studies using mononuclear cells derived from bone marrow, these studies did not show clear effectiveness $(71,72)$. Few studies have described the transplantation of hPSC-CMs using these administration methods.

\section{OTHER ISSUES TO BE SOLVED FOR CARDIAC REGENERATIVE THERAPY}

\section{Arrhythmia After Transplantation of hPSC-CMs}

One of the problems of transplanting hPSC-CMs into the myocardium is the appearance of arrhythmias, such as ventricular tachycardia (VT) $(55-59,62,73)$. In many cases, arrhythmia appeared $\sim 1$ month after transplantation and subsided spontaneously. A study using electrical mapping and pacing suggested that the mechanism of VT after transplantation was not macro-reentry but automaticity (62). Initial hPSCCMs were electrophysiologically immature. For example, the resting membrane potential is less hyperpolarized, and the upstroke velocity of the action potential is much slower in immature hPSC-CMs than in mature ventricular CMs (74). The immaturity of hPSC-CMs, and contamination of pacemaker cells and non-CMs might be responsible for arrhythmia. 
Nakamura et al. reported that a combination of amiodarone and ivabradine could effectively suppress cardiac death and unstable arrhythmia, although drug treatment does not abrogate arrhythmia completely (73).

\section{Immune Rejection}

Another problem with hPSC-CM transplantation is immune rejection. In the present state, allotransplantation is considered favorable because it is necessary to use hPSCs with proven quality and safety. Immunosuppressants should be used to reduce immune rejection. The optimal regimen of immunosuppressants after transplantation of hPSC-CMs is not yet known, although the regimen for patients who undergo HT is helpful. In addition, we should match the hPSC-major histocompatibility complex (MHC) and recipient-MHC in order to reduce the severity of rejection. This is a concern for patients who do not have MHC-compatible hPSCs. Hypoimmunogenic hPSCs are being developed using genetic modification to enable allotransplantation in patients with multiple types of MHCs. For example, hPSCs that are deficient in HLA-I via disruption of beta 2 -microglobulin can evade recognition of recipient $\mathrm{T}$ cells, but they can be attacked by NK cells (75). Therefore, it is necessary to develop hypoimmunogenic hPSCs that can evade the recognition of both T and NK cells. For example, hPSCs that are deficient in HLA-I and HLA-II and express CD47 (76), and hPSCs that are deficient in HLA-A and HLA-B and retain HLA-C (77), can evade the attack of both T cells and NK cells. Because these studies used CRISPR/Cas9 technology, the safety of hPSCs that are genetically modified using this technology should be evaluated.

\section{FUTURE PERSPECTIVES OF CARDIAC REGENERATIVE THERAPY}

Many problems are gradually being solved to achieve cardiac regenerative therapy. Menasché et al. reported that they

\section{REFERENCES}

1. The Japanese Society for Heart Transplantation. The Registry Report of Japanese Heart Transplantation-2020. Ishoku. (2020) 55:261-9. doi: 10.11386/jst.55.3_261

2. Thomson JA, Itskovitz-Eldor J, Shapiro SS, Waknitz MA, Swiergiel JJ, Marshall VS, et al. Embryonic stem cell lines derived from human blastocysts. Science. (1998) 282:1145-7. doi: 10.1126/science.282.5391.1145

3. Takahashi K, Yamanaka S. Induction of pluripotent stem cells from mouse embryonic and adult fibroblast cultures by defined factors. Cell. (2006) 126:663-76. doi: 10.1016/j.cell.2006.07.024

4. Takahashi K, Tanabe K, Ohnuki M, Narita M, Ichisaka T, Tomoda K, et al. Induction of pluripotent stem cells from adult human fibroblasts by defined factors. Cell. (2007) 131:861-72. doi: 10.1016/j.cell.2007.11.019

5. Murry CE, Reinecke H, Pabon LM. Regeneration gaps: observations on stem cells and cardiac repair. $J A m$ Coll Cardiol. (2006) 47:1777-85. doi: 10.1016/j.jacc.2006. 02.002

6. Nakamura T, Sano M, Songyang Z, Schneider MD. A Wnt- and beta -catenindependent pathway for mammalian cardiac myogenesis. Proc Natl Acad Sci USA. (2003) 100:5834-9. doi: 10.1073/pnas.0935626100 transplanted $\sim 8.2$ million hESC-derived cardiovascular progenitors embedded in a fibrin patch onto the epicardium of HF patients and supported their safety (78). Our group is planning to conduct clinical trials on cardiac regenerative therapy using hPSC-CMs. Regarding post-transplantation arrhythmia, the best regimen of antiarrhythmic drugs and the best hPSC-CM status, for example, purity, maturity, and homogeneity should be further evaluated, although the mechanism and duration of arrhythmia, and the effectiveness of drugs on arrhythmia are being studied recently. We will conduct allotransplantation of hPSC-CMs whose MHC will be matched with recipient-MHC in addition to the administration of immunosuppressants. Hypoimmunogenic hPSCs have been developed through genetic modification. In the future, the clinical application of autotransplantation is expected to materialize if high-quality patient-derived hPSCs can be produced rapidly. Although further studies are required, safe and effective cardiac regenerative therapy will be realized in the near future.

\section{AUTHOR CONTRIBUTIONS}

YS and ST wrote the original manuscript. YM, YK, HK, KF, and ST reviewed and edited the manuscript. YS, KF, and ST acquired funding. All authors contributed to the article and approved the submitted version.

\section{FUNDING}

This work was supported by Projects for Technological Development, Research Center Network for Realization of Regenerative Medicine by Japan, the Japan Agency for Medical Research and Development (AMED) grant 20bm0404023h0003 to ST, the Japan Society for the Promotion of Science (JSPS) KAKENHI $20 \mathrm{H} 03768$ to ST, and Grant-in-Aid for JSPS Fellows 21J10680 to YS.
7. Ladd AN, Yatskievych TA, Antin PB. Regulation of avian cardiac myogenesis by activin/TGFbeta and bone morphogenetic proteins. Dev Biol. (1998) 204:407-19. doi: 10.1006/dbio.1998.9094

8. Naito AT, Shiojima I, Akazawa H, Hidaka K, Morisaki T, Kikuchi A, et al. Developmental stage-specific biphasic roles of Wnt/beta-catenin signaling in cardiomyogenesis and hematopoiesis. Proc Natl Acad Sci USA. (2006) 103:19812-7. doi: 10.1073/pnas.0605768103

9. Ueno S, Weidinger G, Osugi T, Kohn AD, Golob JL, Pabon L, et al. Biphasic role for Wnt/beta-catenin signaling in cardiac specification in zebrafish and embryonic stem cells. Proc Natl Acad Sci USA. (2007) 104:968590. doi: 10.1073/pnas.0702859104

10. Laflamme MA, Chen KY, Naumova AV, Muskheli V, Fugate JA, Dupras SK, et al. Cardiomyocytes derived from human embryonic stem cells in prosurvival factors enhance function of infarcted rat hearts. Nat Biotechnol. (2007) 25:1015-24. doi: 10.1038/nbt1327

11. Lian X, Hsiao C, Wilson G, Zhu K, Hazeltine LB, Azarin SM, et al. Robust cardiomyocyte differentiation from human pluripotent stem cells via temporal modulation of canonical Wnt signaling. Proc Natl Acad Sci USA. (2012) 109:E1848-57. doi: 10.1073/pnas.1200250109

12. Hentze H, Soong PL, Wang ST, Phillips BW, Putti TC, Dunn NR. Teratoma formation by human embryonic stem cells: evaluation of 
essential parameters for future safety studies. Stem Cell Res. (2009) 2:198210. doi: 10.1016/j.scr.2009.02.002

13. Fong CY, Peh GS, Gauthaman K, Bongso A. Separation of SSEA-4 and TRA-1-60 labelled undifferentiated human embryonic stem cells from a heterogeneous cell population using magnetic-activated cell sorting (MACS) and fluorescence-activated cell sorting (FACS). Stem Cell Rev Rep. (2009) 5:72-80. doi: 10.1007/s12015-009-9054-4

14. Tang C, Lee AS, Volkmer JP, Sahoo D, Nag D, Mosley AR, et al. An antibody against SSEA-5 glycan on human pluripotent stem cells enables removal of teratoma-forming cells. Nat Biotechnol. (2011) 29:82934. doi: 10.1038/nbt.1947

15. Wang YC, Nakagawa M, Garitaonandia I, Slavin I, Altun G, Lacharite RM, et al. Specific lectin biomarkers for isolation of human pluripotent stem cells identified through array-based glycomic analysis. Cell Res. (2011) 21:155163. doi: $10.1038 /$ cr.2011.148

16. Dubois NC, Craft AM, Sharma P, Elliott DA, Stanley EG, Elefanty AG, et al. SIRPA is a specific cell-surface marker for isolating cardiomyocytes derived from human pluripotent stem cells. Nat Biotechnol. (2011) 29:10118. doi: 10.1038/nbt.2005

17. Hattori F, Chen H, Yamashita H, Tohyama S, Satoh YS, Yuasa S, et al. Nongenetic method for purifying stem cell-derived cardiomyocytes. Nat Methods. (2010) 7:61-6. doi: 10.1038/nmeth.1403

18. Kuang Y, Miki K, Parr CJC, Hayashi K, Takei I, Li J, et al. Efficient, selective removal of human pluripotent stem cells via ecto-alkaline phosphatasemediated aggregation of synthetic peptides. Cell Chem Biol. (2017) 24:68594.e4. doi: 10.1016/j.chembiol.2017.04.010

19. Lee MO, Moon SH, Jeong HC, Yi JY, Lee TH, Shim SH, et al. Inhibition of pluripotent stem cell-derived teratoma formation by small molecules. Proc Natl Acad Sci USA. (2013) 110:E3281-90. doi: 10.1073/pnas.1303669110

20. Tateno H, Onuma $\mathrm{Y}$, Ito $\mathrm{Y}$, Minoshima F, Saito S, Shimizu M, et al. Elimination of tumorigenic human pluripotent stem cells by a recombinant lectin-toxin fusion protein. Stem Cell Rep. (2015) 4:811-20. doi: 10.1016/j.stemcr.2015.02.016

21. Ben-David U, Nudel N, Benvenisty N. Immunologic and chemical targeting of the tight-junction protein Claudin-6 eliminates tumorigenic human pluripotent stem cells. Nat Commun. (2013) 4:1992. doi: $10.1038 /$ ncomms 2992

22. Tohyama S, Hattori F, Sano M, Hishiki T, Nagahata Y, Matsuura T, et al. Distinct metabolic flow enables large-scale purification of mouse and human pluripotent stem cell-derived cardiomyocytes. Cell Stem Cell. (2013) 12:12737. doi: 10.1016/j.stem.2012.09.013

23. Tohyama S, Fujita J, Hishiki T, Matsuura T, Hattori F, Ohno R, et al. Glutamine oxidation is indispensable for survival of human pluripotent stem cells. Cell Metab. (2016) 23:663-74. doi: 10.1016/j.cmet.2016.03.001

24. Shiraki N, Shiraki Y, Tsuyama T, Obata F, Miura M, Nagae G, et al. Methionine metabolism regulates maintenance and differentiation of human pluripotent stem cells. Cell Metab. (2014) 19:780-94. doi: 10.1016/j.cmet.2014.03.017

25. Ben-David U, Gan QF, Golan-Lev T, Arora P, Yanuka O, Oren YS, et al. Selective elimination of human pluripotent stem cells by an oleate synthesis inhibitor discovered in a high-throughput screen. Cell Stem Cell. (2013) 12:167-79. doi: 10.1016/j.stem.2012.11.015

26. Tanosaki S, Tohyama S, Fujita J, Someya S, Hishiki T, Matsuura T, et al. Fatty acid synthesis is indispensable for survival of human pluripotent stem cells. iScience. (2020) 23:101535. doi: 10.1016/j.isci.2020.101535

27. Okada M, Tada Y, Seki T, Tohyama S, Fujita J, Suzuki T, et al. Selective elimination of undifferentiated human pluripotent stem cells using pluripotent state-specific immunogenic antigen Glypican-3. Biochem Biophys Res Commun. (2019) 511:711-7. doi: 10.1016/j.bbrc.2019.02.094

28. Parr CJ, Katayama S, Miki K, Kuang Y, Yoshida Y, Morizane A, et al. MicroRNA-302 switch to identify and eliminate undifferentiated human pluripotent stem cells. Sci Rep. (2016) 6:32532. doi: 10.1038/srep32532

29. Chen VC, Ye J, Shukla P, Hua G, Chen D, Lin Z, et al. Development of a scalable suspension culture for cardiac differentiation from human pluripotent stem cells. Stem Cell Res. (2015) 15:365-75. doi: 10.1016/j.scr.2015.08.002

30. Kempf H, Olmer R, Kropp C, Rückert M, Jara-Avaca M, Robles-Diaz D, et al. Controlling expansion and cardiomyogenic differentiation of human pluripotent stem cells in scalable suspension culture. Stem Cell Rep. (2014) 3:1132-46. doi: 10.1016/j.stemcr.2014.09.017

31. Fonoudi H, Ansari H, Abbasalizadeh S, Larijani MR, Kiani S, Hashemizadeh $S$, et al. A universal and robust integrated platform for the scalable production of human cardiomyocytes from pluripotent stem cells. Stem Cells Transl Med. (2015) 4:1482-94. doi: 10.5966/sctm.2014-0275

32. Halloin C, Schwanke K, Löbel W, Franke A, Szepes M, Biswanath S, et al. Continuous WNT control enables advanced hPSC cardiac processing and prognostic surface marker identification in chemically defined suspension culture. Stem Cell Rep. (2019) 13:366-79. doi: 10.1016/j.stemcr.2019.06.004

33. Ting S, Chen A, Reuveny S, Oh S. An intermittent rocking platform for integrated expansion and differentiation of human pluripotent stem cells to cardiomyocytes in suspended microcarrier cultures. Stem Cell Res. (2014) 13:202-13. doi: 10.1016/j.scr.2014.06.002

34. Ting S, Lam A, Tong G, Chen A, Wei H, Wu J, et al. Meticulous optimization of cardiomyocyte yields in a 3-stage continuous integrated agitation bioprocess. Stem Cell Res. (2018) 31:161-73. doi: 10.1016/j.scr.2018.07.020

35. Tohyama S, Fujita J, Fujita C, Yamaguchi M, Kanaami S, Ohno R, et al. Efficient large-scale 2D culture system for human induced pluripotent stem cells and differentiated cardiomyocytes. Stem Cell Rep. (2017) 9:140614. doi: 10.1016/j.stemcr.2017.08.025

36. Rupert CE, Irofuala C, Coulombe KLK. Practical adoption of state-ofthe-art hiPSC-cardiomyocyte differentiation techniques. PLoS ONE. (2020) 15:e0230001. doi: 10.1371/journal.pone.0230001

37. Tohyama S, Fukuda K. Safe and effective cardiac regenerative therapy with human-induced pluripotent stem cells: how should we prepare pure cardiac myocytes? Circ Res. (2017) 120:155860. doi: 10.1161/CIRCRESAHA.116.310328

38. Lopaschuk GD, Jaswal JS. Energy metabolic phenotype of the cardiomyocyte during development, differentiation, and postnatal maturation. J Cardiovasc Pharmacol. (2010) 56:130-40. doi: 10.1097/FJC.0b013e3181e74a14

39. Sugiura Y, Katsumata Y, Sano M, Honda K, Kajimura M, Fukuda K, et al. Visualization of in vivo metabolic flows reveals accelerated utilization of glucose and lactate in penumbra of ischemic heart. Sci Rep. (2016) 6:32361.

40. Olivetti G, Capasso JM, Sonnenblick EH, Anversa P. Side-to-side slippage of myocytes participates in ventricular wall remodeling acutely after myocardial infarction in rats. Circ Res. (1990) 67:23-34. doi: 10.1161/01.RES.67.1.23

41. Caulfield JB, Leinbach R, Gold H. The relationship of myocardial infarct size and prognosis. Circulation. (1976) 53:I141-4.

42. Hemmi N, Tohyama S, Nakajima K, Kanazawa H, Suzuki T, Hattori F, et al. A massive suspension culture system with metabolic purification for human pluripotent stem cell-derived cardiomyocytes. Stem Cells Transl Med. (2014) 3:1473-83. doi: 10.5966/sctm.2014-0072

43. Miyazaki T, Futaki S, Hasegawa K, Kawasaki M, Sanzen N, Hayashi M, et al. Recombinant human laminin isoforms can support the undifferentiated growth of human embryonic stem cells. Biochem Biophys Res Commun. (2008) 375:27-32. doi: 10.1016/j.bbrc.2008.07.111

44. Chen G, Gulbranson DR, Hou Z, Bolin JM, Ruotti V, Probasco MD, et al. Chemically defined conditions for human iPSC derivation and culture. Nat Methods. (2011) 8:424-9. doi: 10.1038/nmeth.1593

45. Klim JR, Li L, Wrighton PJ, Piekarczyk MS, Kiessling LL. A defined glycosaminoglycan-binding substratum for human pluripotent stem cells. Nat Methods. (2010) 7:989-94. doi: 10.1038/nmeth.1532

46. Melkoumian Z, Weber JL, Weber DM, Fadeev AG, Zhou Y, Dolley-Sonneville $\mathrm{P}$, et al. Synthetic peptide-acrylate surfaces for long-term self-renewal and cardiomyocyte differentiation of human embryonic stem cells. Nat Biotechnol. (2010) 28:606-10. doi: $10.1038 /$ nbt.1629

47. Someya S, Tohyama S, Kameda K, Tanosaki S, Morita Y, Sasaki K, et al. Tryptophan metabolism regulates proliferative capacity of human pluripotent stem cells. iScience. (2021) 24:102090. doi: 10.1016/j.isci.2021.102090

48. Kawamura M, Miyagawa S, Miki K, Saito A, Fukushima S, Higuchi $\mathrm{T}$, et al. Feasibility, safety, and therapeutic efficacy of human induced pluripotent stem cell-derived cardiomyocyte sheets in a porcine ischemic cardiomyopathy model. Circulation. (2012) 126:S29-37. doi: 10.1161/CIRCULATIONAHA.111.084343

49. Gerbin KA, Yang X, Murry CE, Coulombe KL. Enhanced electrical integration of engineered human myocardium via intramyocardial 
versus epicardial delivery in infarcted rat hearts. PLoS ONE. (2015) 10:e0131446. doi: 10.1371/journal.pone.0131446

50. Higuchi T, Miyagawa S, Pearson JT, Fukushima S, Saito A, Tsuchimochi H, et al. Functional and electrical integration of induced pluripotent stem cellderived cardiomyocytes in a myocardial infarction rat heart. Cell Transplant. (2015) 24:2479-89. doi: 10.3727/096368914X685799

51. Li J, Minami I, Shiozaki M, Yu L, Yajima S, Miyagawa S, et al. Human pluripotent stem cell-derived cardiac tissue-like constructs for repairing the infarcted myocardium. Stem Cell Rep. (2017) 9:154659. doi: 10.1016/j.stemcr.2017.09.007

52. Weinberger F, Breckwoldt K, Pecha S, Kelly A, Geertz B, Starbatty $\mathrm{J}$, et al. Cardiac repair in guinea pigs with human engineered heart tissue from induced pluripotent stem cells. Sci Transl Med. (2016) 8:363ra148. doi: 10.1126/scitranslmed.aaf8781

53. Querdel E, Reinsch M, Castro L, Köse D, Bähr A, Reich S, et al. Human engineered heart tissue patches remuscularize the injured heart in a dose-dependent manner. Circulation. (2021) 143:1991-2006. doi: 10.1161/CIRCULATIONAHA.120.047904

54. Suzuki K, Miyagawa S, Liu L, Kawamura T, Li J, Qu X, et al. Therapeutic efficacy of large aligned cardiac tissue derived from induced pluripotent stem cell in a porcine ischemic cardiomyopathy model. J Heart Lung Transplant. (2021) 40:767-77. doi: 10.1016/j.healun.2021.04.010

55. Shiba Y, Fernandes S, Zhu WZ, Filice D, Muskheli V, Kim J, et al. Human EScell-derived cardiomyocytes electrically couple and suppress arrhythmias in injured hearts. Nature. (2012) 489:322-5. doi: 10.1038/nature11317

56. Chong JJ, Yang X, Don CW, Minami E, Liu YW, Weyers JJ, et al. Human embryonic-stem-cell-derived cardiomyocytes regenerate non-human primate hearts. Nature. (2014) 510:273-7. doi: 10.1038/nature13233

57. Shiba Y, Gomibuchi T, Seto T, Wada Y, Ichimura H, Tanaka Y, et al. Allogeneic transplantation of iPS cell-derived cardiomyocytes regenerates primate hearts. Nature. (2016) 538:388-91. doi: 10.1038/nature19815

58. Liu YW, Chen B, Yang X, Fugate JA, Kalucki FA, Futakuchi-Tsuchida A, et al. Human embryonic stem cell-derived cardiomyocytes restore function in infarcted hearts of non-human primates. Nat Biotechnol. (2018) 36:597605. doi: 10.1038/nbt.4162

59. Kawaguchi S, Soma Y, Nakajima K, Kanazawa H, Tohyama S, Tabei R, et al. Intramyocardial transplantation of human iPS cell-derived cardiac spheroids improves cardiac function in heart failure animals. JACC Basic Transl Sci. (2021) 6:239-54. doi: 10.1016/j.jacbts.2020.11.017

60. Cho GS, Lee DI, Tampakakis E, Murphy S, Andersen P, Uosaki $\mathrm{H}$, et al. Neonatal transplantation confers maturation of PSC-derived cardiomyocytes conducive to modeling cardiomyopathy. Cell Rep. (2017) 18:571-82. doi: 10.1016/j.celrep.2016.12.040

61. Kadota S, Pabon L, Reinecke H, Murry CE. In vivo maturation of human induced pluripotent stem cell-derived cardiomyocytes in neonatal and adult rat hearts. Stem Cell Reports. (2017) 8:278-89. doi: 10.1016/j.stemcr.2016.10.009

62. Romagnuolo R, Masoudpour H, Porta-Sánchez A, Qiang B, Barry J, Laskary A, et al. Human embryonic stem cell-derived cardiomyocytes regenerate the infarcted pig heart but induce ventricular tachyarrhythmias. Stem Cell Rep. (2019) 12:967-81. doi: 10.1016/j.stemcr.2019.04.005

63. Hattan N, Kawaguchi H, Ando K, Kuwabara E, Fujita J, Murata M, et al. Purified cardiomyocytes from bone marrow mesenchymal stem cells produce stable intracardiac grafts in mice. Cardiovasc Res. (2005) 65:33444. doi: 10.1016/j.cardiores.2004.10.004

64. Nakajima K, Fujita J, Matsui M, Tohyama S, Tamura N, Kanazawa H, et al. Gelatin hydrogel enhances the engraftment of transplanted cardiomyocytes and angiogenesis to ameliorate cardiac function after myocardial infarction. PLoS ONE. (2015) 10:e0133308. doi: 10.1371/journal.pone.0133308

65. Lee AS, Inayathullah M, Lijkwan MA, Zhao X, Sun W, Park S, et al. Prolonged survival of transplanted stem cells after ischaemic injury via the slow release of pro-survival peptides from a collagen matrix. Nat Biomed Eng. (2018) 2:104-13. doi: 10.1038/s41551-018-0191-4

66. Tabei R, Kawaguchi S, Kanazawa H, Tohyama S, Hirano A, Handa N, et al. Development of a transplant injection device for optimal distribution and retention of human induced pluripotent stem cell-derived cardiomyocytes. J Heart Lung Transplant. (2019) 38:203-14. doi: 10.1016/j.healun.2018.11.002

67. Park SJ, Kim RY, Park BW, Lee S, Choi SW, Park JH, et al. Dual stem cell therapy synergistically improves cardiac function and vascular regeneration following myocardial infarction. Nat Commun.

(2019) 10:3123. doi: 10.1038/s41467-019-11091-2

68. Bargehr J, Ong LP, Colzani M, Davaapil H, Hofsteen P, Bhandari S, et al. Epicardial cells derived from human embryonic stem cells augment cardiomyocyte-driven heart regeneration. Nat Biotechnol. (2019) 37:895906. doi: 10.1038/s41587-019-0197-9

69. Sun X, Wu J, Qiang B, Romagnuolo R, Gagliardi M, Keller G, et al. Transplanted microvessels improve pluripotent stem cell-derived cardiomyocyte engraftment and cardiac function after infarction in rats. Sci Transl Med. (2020) 12:eaax2992. doi: 10.1126/scitranslmed.aax2992

70. Funakoshi S, Fernandes I, Mastikhina O, Wilkinson D, Tran $\mathrm{T}$, Dhahri W, et al. Generation of mature compact ventricular cardiomyocytes from human pluripotent stem cells. Nat Commun. (2021) 12:3155. doi: 10.1038/s41467-021-23329-z

71. Perin EC, Willerson JT, Pepine CJ, Henry TD, Ellis SG, Zhao DX, et al. Effect of transendocardial delivery of autologous bone marrow mononuclear cells on functional capacity, left ventricular function, and perfusion in chronic heart failure: the FOCUS-CCTRN trial. JAMA. (2012) 307:171726. doi: 10.1001/jama.2012.418

72. Traverse JH, Henry TD, Pepine CJ, Willerson JT, Zhao DX, Ellis SG, et al. Effect of the use and timing of bone marrow mononuclear cell delivery on left ventricular function after acute myocardial infarction: the TIME randomized trial. JAMA. (2012) 308:2380-9. doi: 10.1001/jama.2012.28726

73. Nakamura K, Neidig LE, Yang X, Weber GJ, El-Nachef D, Tsuchida $\mathrm{H}$, et al. Pharmacologic therapy for engraftment arrhythmia induced by transplantation of human cardiomyocytes. Stem Cell Reports. (2021) 16:247387. doi: 10.1016/j.stemcr.2021.08.005

74. Karbassi E, Fenix A, Marchiano S, Muraoka N, Nakamura K, Yang $\mathrm{X}$, et al. Cardiomyocyte maturation: advances in knowledge and implications for regenerative medicine. Nat Rev Cardiol. (2020) 17:341-59. doi: 10.1038/s41569-019-0331-x

75. Lu P, Chen J, He L, Ren J, Chen H, Rao L, et al. Generating hypoimmunogenic human embryonic stem cells by the disruption of beta 2microglobulin. Stem Cell Rev Rep. (2013) 9:806-13. doi: 10.1007/s12015-0139457-0

76. Deuse T, Hu X, Gravina A, Wang D, Tediashvili G, De C, et al. Hypoimmunogenic derivatives of induced pluripotent stem cells evade immune rejection in fully immunocompetent allogeneic recipients. Nat Biotechnol. (2019) 37:252-8. doi: 10.1038/s41587-019-0016-3

77. Xu H, Wang B, Ono M, Kagita A, Fujii K, Sasakawa N, et al. Targeted disruption of HLA genes via CRISPR-Cas9 generates iPSCs with enhanced immune compatibility. Cell Stem Cell. (2019) 24:56678.e7. doi: 10.1016/j.stem.2019.02.005

78. Menasché P, Vanneaux V, Hagège A, Bel A, Cholley B, Cacciapuoti I, et al. Human embryonic stem cell-derived cardiac progenitors for severe heart failure treatment: first clinical case report. Eur Heart J. (2015) 36:20117. doi: 10.1093/eurheartj/ehv189

Conflict of Interest: KF is a co-founder and CEO of Heartseed, Inc. ST was an advisor of Heartseed, Inc. ST, HK, and KF owned equity in Heartseed, Inc.

The remaining authors declare that the research was conducted in the absence of any commercial or financial relationships that could be construed as a potential conflict of interest.

Publisher's Note: All claims expressed in this article are solely those of the authors and do not necessarily represent those of their affiliated organizations, or those of the publisher, the editors and the reviewers. Any product that may be evaluated in this article, or claim that may be made by its manufacturer, is not guaranteed or endorsed by the publisher.

Copyright (c) 2021 Soma, Morita, Kishino, Kanazawa, Fukuda and Tohyama. This is an open-access article distributed under the terms of the Creative Commons Attribution License (CC BY). The use, distribution or reproduction in other forums is permitted, provided the original author(s) and the copyright owner(s) are credited and that the original publication in this journal is cited, in accordance with accepted academic practice. No use, distribution or reproduction is permitted which does not comply with these terms. 